Military Technical College

Kobry El-Kobba

Cairo, Egypt

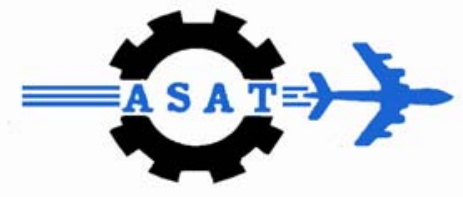

12-th International Conference

on

Aerospace Sciences \&

Aviation Technology

\title{
DOWNSTREAM INSTABILITY OF VISCOELASTIC FLOW PAST A CYLINDER
}

\author{
H. KAMAL ${ }^{\S *}$, H. NAJI*, L. THAIS* \& G. MOMPEAN*
}

\begin{abstract}
This work aims at simulating two-dimensional viscoelastic incompressible fluid flow past a non-confined cylinder. The finite volume method is used to descritize the governing equations written in the generalized orthogonal coordinate system. For the viscoelastic constitutive equation, two models are studied; the Oldryod-B model and the Phan-Thien-Tanner model PTT model. The quadratic scheme QUICK is employed to evaluate the convection terms. The terms of diffusion, advection and acceleration are treated explicitly. The Elastic Viscous Split Stresses (EVSS) scheme is used to decompose the stress tensor to enhance the stability of computations.

The obtained results indicate that, for Newtonian flow, the onset of von Karman street occurs at Reynolds number $R e \geq 47$. Upon the onset of vortex shedding the downstream instability zone is elongated and the shedding frequency is reduced. The PTT model proves more stability of computations and reached higher Deborah numbers.
\end{abstract}

\section{KEY WORDS}

Viscoelastic Fluids, Generalized Orthogonal Coordinates, Flow past a Cylinder

\footnotetext{
$\S$ Egyptian Armed Forces

* Laboratoire de Mécanique de Lille, UMR-CNRS 8107

Université des Sciences et Technologies de Lille, Polytech'Lille - Cité Scientifique, 59655 Villeneuve d'Ascq Cedex, France Hossam.Kamal@polytech-lille.fr
} 


\section{NOMENCLATURE}

Latin symbols:
A
$\hat{a}_{i}$
cylinder radius.
$\bar{C}_{b p}$
$\bar{C}_{d}$
covariant base vectors .
$\bar{C}_{l}$
average base pressure coefficient.
average drag coefficient.
$D_{i j} \equiv(\mathbf{D})$
average lift coefficient.
$f$
the rate of deformation tensor (matrix).
G
frequency of vortex shedding.
$g_{i j}$
function representing the elongational viscosity.
$H_{i}{ }^{j}$
metric tensor .
$h_{i}$
coordinate variation term.
$J$
scale factors.
$\mathcal{L}=\nabla u_{j}-\zeta D_{i j}$.
$n$
Jacobian of coordinate transformation.
$p \quad$ pressure field.
$\vec{r} \quad$ position vector.
Str Strouhal number.
$S_{\Phi} \quad$ source term.
$T_{i j} \quad$ viscous stress tensor.
$t$ time.
$U_{0} \quad$ free-upstream velocity.
$u_{i} \quad$ velocity vector.
$V_{i} \quad$ physical components of velocity.
$\vec{x}\left(x_{1}, x_{2}\right) \quad$ Cartesian coordinate system.

Greek symbols:

$\beta$

$\Gamma$

$\dot{\gamma}$

$\delta_{i j}$

$\varepsilon$

$\zeta$

$\eta_{0}$

$\eta_{m}$

$\eta_{m 0}$

$\eta_{N}$

$\Theta$

$\Lambda$

$\lambda$

$\vec{\xi}\left(\xi_{1}, \xi_{2}\right)$ retardation ratio.

time parameter.

generalized shear rate.

Kroneker delta.

material parameter related to its elongation behaviour.

material parameter related to its elongation behaviour.

the zero-shear rate viscosity of the fluid.

molecular contributed viscosity.

zero-shear rate molecular contributed viscosity.

solvent viscosity.

coefficients of generalized transport equation.

coefficients of generalized transport equation.

relaxation time.

physical components. 


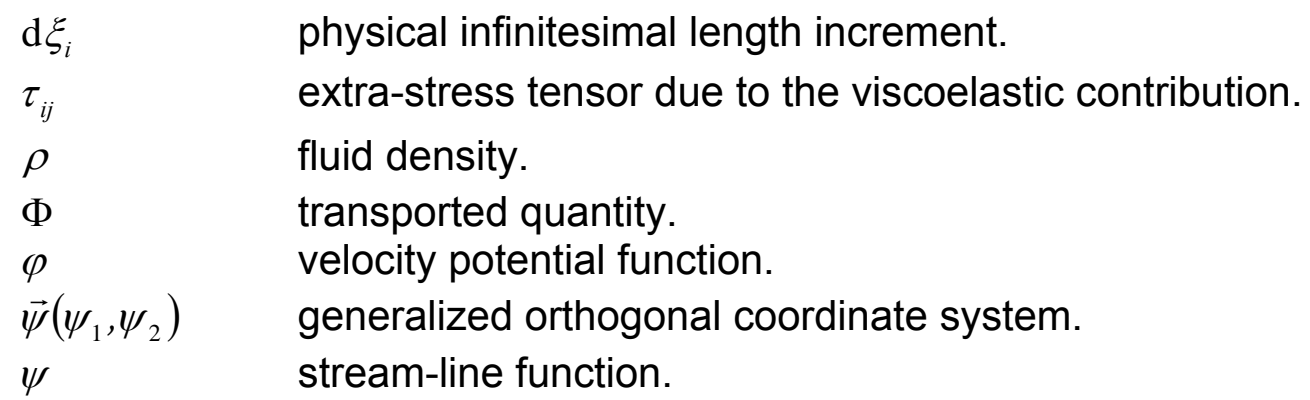

Operators:

$\nabla_{i}() \quad$ divergence operator.

\section{INTRODUCTION}

Viscoelastic fluids are one class of non-Newtonian fluids. They are also referred to as polymeric liquids, meaning that they contain some fraction of high molecular weight substance. The viscosity of a polymer solution is usually not a material constant, but depends on the flow state of the liquid, namely the local shear rate. When a viscoelastic material is suddenly stretched and then kept fixed in that state, the force it exerts will first rise sharply and then decrease with a characteristic relaxation time. This effect is also the origin of the term "viscoelastic". An elastic body would hold the tension once developed, whereas a viscous fluid would only exert a force during the short stretching period.

The understanding of such a flow is required for important engineering applications and has attracted a great deal of attention in the literature. This simplified model configuration is employed to study important fluid mechanical phenomena including drag characteristics and the detailed kinematics such as wake structure, vortex shedding frequency, etc. Similarly, this information is frequently needed for the design of structures exposed to fluid flow (such as off-shore pipelines, piers and supports, heat exchangers, ...etc.). [1].

Cadot \& Lebey [2] visualized the wake behind a circular cylinder and concluded that a drastic change of the shape of the wake of viscoelastic solutions is observed compared to that observed for water injections. They also remarked that the aspect ratio of the wake is decreased, the wavelength of the vortices is increased, and a large region of slow fluid motion is developed behind the cylinder indicating a delay of the shear roll-up and thus an inhibition of the shear instability. Pipe \& Monkewtiz [3] have characterized the influence of small amounts of fluid elasticity on the 2-D laminar vortex shedding instability and the effects on the fluctuating and timeaverage velocity fields.

The Phan-Thien-Tanner (PTT) model represents the promising simple member of the family of viscoelastic differential constitutive equations. Dou \& Phan-Thien $[4,5]$ studied the flow past a confined cylinder but foucused on the parallisation. They used the Oldroyd-B model (extracted from the same equation of PTT) and added discrete elastic viscous split stress (DEVSS) formulation together with an independent interpolation of the vorticity. Phan-Thien \& Dou [6] investigated the drag coefficient of confined cylinder and found that the drag coefficient for Upper convected Maxwell (UCM) fluid firstly decreases with the increasing Deborah number at low values and then seems to tend to be a constant at high values of Deborah number, while for PTT 
fluid it decreases rapidly with the increasing Deborah number at low Deborah, and then slowly decreases with Deborah at high Deborah number.

The generalized orthogonal coordinates offer an effective solution for complex geometry fluid flow. Pope [7] proposed a methode for adapting the generalized orthogonal coordinates to simulate the turbulent flow through a diffuser. This method proves its efficiency.

In this work, the two-dimensional viscoelastic incompressible fluid flow past a cylinder is simulated using the finite volume method to descritize the governing equations in generalized orthogonal coordinate system. Two viscoelastic models are studied; the Oldryod-B model and the Phan-Thien-Tanner (PTT) model.

\section{GOVERNING EQUATIONS}

\section{Governing equations in Cartesian coordinate system}

The fluid flow past a cylinder is governed by the mass and momentum conservation equations:

$$
\begin{aligned}
& \frac{\partial u_{i}}{\partial x_{i}}=0 \\
& \rho\left(\frac{\partial u_{i}}{\partial t}+u_{j} \frac{\partial u_{i}}{\partial x_{j}}\right)=-\frac{\partial p}{\partial x_{j}}+\frac{\partial T_{i j}}{\partial x_{j}}
\end{aligned}
$$

For the viscoelastic fluids, the viscous stress tensor is decomposed into two parts; the Newtonian stress tensor $2 \eta_{N} D_{i j}$ (with $\eta_{N}$ usually, but not necessarily, the solvent viscosity) and the extra-stress tensor due to the viscoelastic contribution $\tau_{i j}$

$$
\begin{aligned}
& T_{i j}=2 \eta_{N} D_{i j}+\tau_{i j} \\
& \mathbf{D}=\frac{1}{2}\left(\nabla \mathbf{U}+(\nabla \mathbf{U})^{\mathrm{T}}\right)
\end{aligned}
$$

The viscoelastic extra-stress tensor is related to the velocity field through the constitutive equation:

$$
G \tau_{i j}+\lambda\left(\frac{\partial \tau_{i j}}{\partial t}+u_{i} \cdot \frac{\partial \tau_{i j}}{\partial x_{j}}-\mathcal{L} \tau_{i j}-\tau_{i j} \mathcal{L}^{T}\right)=2 \eta_{m} D_{i j}
$$

where $G=\exp \left(\lambda \varepsilon / \eta_{m 0} \operatorname{Tr}\left\langle\tau_{i j}\right\rangle\right)$, and $\mathcal{L}=\nabla u_{j}-\zeta D_{i j}$. The two viscosities $\eta_{\mathrm{m}}$ and $\eta_{m 0}$ are related by:

$$
\eta_{m}=\eta_{m 0} \mu \quad, \quad \mu=\left(\frac{1+\zeta(2-\zeta) \lambda^{2} \dot{\gamma}^{2}}{\left(1+\Gamma^{2} \dot{\gamma}^{2}\right)^{(1-n) / 2}}\right)
$$

where $\dot{\gamma}=\sqrt{2 \operatorname{Tr}\left\langle D_{i j}\right\rangle}$ is the generalized shear rate, and $\Gamma$ is a time parameter assumed to be $\Gamma=\lambda$.

The constitutive equation (5)can now be rewritten as a transport equation of $\tau_{i j}$ :

$$
\lambda\left(\frac{\partial \tau_{i j}}{\partial t}+\frac{\partial}{\partial x_{j}}\left(u_{i} \tau_{i j}\right)\right)=2 \mu \beta \eta_{0} D_{i j}+\lambda\left(\mathcal{L} \tau_{i j}+\tau_{i j} \mathcal{L}\right)-G \tau_{i j}
$$


where $\eta_{0}=\eta_{N}+\eta_{m 0}$ is the zero-shear rate viscosity of the fluid and $\beta=\eta_{m 0} / \eta_{0}$ is the retardation ratio. According to the values of $\beta, \varepsilon$, and $\zeta$, six models can be defined:

- Newtonian Fluid $\left(\varepsilon=0, \xi=0, \beta=0, \eta_{0}=\eta_{N}\right)$

- Upper Convected Maxwell (UCM) $\left(\varepsilon=0, \xi=0, \beta=1, \eta_{0}=\eta_{m}=\eta_{m 0}\right)$

- Oldroyd-B $\left(\varepsilon=0, \xi=0,0<\beta<1, \eta_{0}=\eta_{m}=\eta_{m 0}\right)$

- $\quad$ simplified PTT (SPTT) $\left(\xi=0, \beta=1, \eta_{0}=\eta_{m}=\eta_{m 0}\right)$

- $\quad$ modified PTT (MPTT) $\left(\beta=1, \eta_{0}=\eta_{m}=\eta_{m 0}\right)$

\section{Governing equations in general orthogonal coordinate system}

For the case of two-dimensional flow, we have the Cartesian coordinate system $\vec{x}\left(x_{1}, x_{2}\right)$ and the generalized orthogonal coordinate system $\vec{\psi}\left(\psi_{1}, \psi_{2}\right)$. The admissible transformation between two coordinate systems is carried out through the Jacobian $J$ which is defined by $J=|\partial \vec{x} / \partial \vec{\psi}|$.

The metric tensor relates distances to the infinitesimal coordinate increments; and is given by $g_{i j}=\hat{a}_{i} \circ \hat{a}_{j}=g_{j i}$ with $\hat{a}_{i}, i=1,2$ are the covariant base vectors defined by $\hat{a}_{i}=\partial \vec{r} / \partial \bar{x}^{i}$ with the position vector $\vec{r}$. The condition of orthogonality implies that the diagonal terms of the metric tensor are nonzero. Its convenient to write $g_{i i}=h_{i}^{2}$ where $h_{i}$ are called the scale factors which represent the ratio of a distance to the coordinate differences.

In order to formulate the governing equations in generalized orthogonal coordinates the methodology described in Pope [7] is applied and the variables are expressed in terms of their physical components $\vec{\xi}\left(\xi_{1}, \xi_{2}\right)$. The advantage of using physical components rather than covariant or contravariant components is that the vectors retain the same dimensions in all directions and in all locations and tat there are no additional terms for the stretching of the coordinate system.

The governing equations can now be rewritten in generalized orthogonal coordinate system as follows:

The continuity equation

$$
\nabla_{i} V_{i}=0
$$

The momentum equation

$$
\rho \frac{\partial V_{i}}{\partial t}+\rho V_{j}\left(\frac{\partial V_{i}}{\partial \xi_{j}}-V_{i} H_{j}{ }^{i}+V_{k} H_{i}{ }^{k} \delta_{i j}\right)=-\frac{\partial p}{\partial \xi_{j}}+\left(\nabla_{i} T_{i j}-T_{i i} H_{i}{ }^{j}+H_{j}{ }^{k} T_{j k}\right)
$$

The constitutive equation

$$
\lambda\left(\frac{\partial \tau_{i j}}{\partial t}+\left(\frac{\partial}{\partial \xi_{j}}\left(u_{i} \tau_{i j}\right)-\left(u_{i} \tau_{i j}\right) H_{j}{ }^{i}+\left(u_{i} \tau_{i j}\right) H_{i}{ }^{k} \delta_{i j}\right)\right)=2 \mu \beta \eta_{0} D_{i j}+\lambda\left(\mathcal{L}_{i j} \tau_{i j}+\tau_{i j} \mathcal{L}_{j i}\right)-G \tau_{i j}(10
$$

where $\mathrm{d} \xi_{i}=h_{i} \mathrm{~d} \psi_{i}$ is the physical infinitesimal length increment, $V_{i}=h_{i} u_{i}$ is the physical components of velocity, $H_{i}{ }^{j} \equiv\left(1 / h_{i} h_{j}\right)\left(\partial h_{i} / \partial h_{i}\right)$ is the coordinate variation term which represents the inverse of the radius of curvature, and $\nabla_{i}() \equiv \partial() / \partial \xi_{i}+\sum_{k \neq i} H_{i}^{k}()$ is the divergence operator. 


\section{NUMERICAL METHOD}

In order to solve the initial value problem defined by the equations (8), (9), and (10) with appropriate boundary conditions, the finite volume method is used. The general form of the transport equation can be used to scribe all the governing equations.

$$
\frac{\partial}{\partial t}(\Lambda \Phi)+\frac{\partial}{\partial x_{j}}\left(\Lambda u_{j} \Phi\right)=\frac{\partial}{\partial x_{j}}\left(\Theta \frac{\partial \Phi}{\partial x_{j}}\right)+S_{\Phi}
$$

where $\Phi$ is the transported quantity which can be scalar, vector component, or tensor. The coefficients $\Theta$ and $\Lambda$ have different meanings depending on the transported variable. The first right-hand side term is the rate of change term, the second one is the convective term, the first left-hand side term is the diffusion term, and the last term $S_{\Phi}$ is the source term which includes all terms that cannot be accommodated in the convective or diffusive terms.

To stabilize the computation of the constitutive equation, the elastic viscous split stress (EVSS) formulation is applied to stabilize numerical scheme by separating explicitly the elastic and viscous stresses.

For the time marching algorithm, the first-order accurate Euler forward explicit scheme is applied obeying the adaptation of Marker and Cell algorithm developed by Mompean and Deville [8].

The spatial discretization is performed in a staggered mesh. The convective terms are evaluated using the second-order accurate "Quadratic Upstream Interpolation Scheme for Convective Kinematics" (QUICK) scheme. The diffusion terms are calculated with the second order accurate centered difference scheme.

The velocity potential function $\varphi=U_{0} x \cdot\left(1+A^{2} /\left(x^{2}+y^{2}\right)\right)$ and the stream-line function $\psi=U_{0} \cdot y \cdot\left(1-A^{2} /\left(x^{2}+y^{2}\right)\right)$ are used to generate the mesh; where $U_{o}$ is the freeupstream velocity and $A$ is the cylinder radius. The generated mesh is optimised at $160 \times 80$ control volumes. Figure 1 represents a zoomed view of the mesh around the cylinder and the extensions of domain limits to guarantee the non-confined case.

For non-confined case, the boundary conditions are considered to be $u_{x}=U_{0}, u_{z}=0, p=p_{\text {atm }}$ at the east of domain, and symmetry boundary conditions for both top and bottom boundaries.

\section{RESULTS AND DISCUSSION}

In our pervious work, Kamal et al. [9], the Newtonian fluid flow past a cylinder was simulated. We found that the profile of the 2-D flow past the cylinder allow to fully identify 3 different vortex shedding regimes:

- $\quad$ Creep regime $(R e<5)$, with no separation from the cylinder.

- Laminar steady regime $(5 \leq R e<47)$, in which two steady symmetrically vortices on each side of the wake are created and as the Reynolds number increases the inertia force increases over the viscous force and the instability region becomes bigger and elongated.

- Laminar vortex shedding regime $(R e \geq 47)$, the two vortices are no longer symmetric or steady and the von Kàrmàn street is established.

The study of 2-D flow is limited to $R e<190$ because after this limit the 3-D calculation is an obligation (cf. Williamson [10]). 
In this work, we continue applying the same procedure but for the laminar incompressible viscoelastic fluid flow. In order to validate our results, three nondimensional quantities are adopted: non-dimensional frequency represented by the Strouhal number $S t r=2 A f / U_{0}$ where $f$ is the frequency of vortex shedding, the average drag coefficient $\bar{C}_{d}$, and the average base pressure coefficient $\bar{C}_{b p}$.

The previous literatures which discussed the flow of viscoelastic fluid past a nonconfined cylinder are rare and, up to our knowledge, the PTT model with the generalized orthogonal coordinates for such a case has not been used before. Hence, in order to validate the present simulation, the results are compared with that of Oliveira [11] who applied the FENE-CR model. Table 1, resumes the comparison of average drag coefficient for both PTT an Oldroyd models versus the results of Oliveira [11]. This comparison shows good agreement of results. The results of PTT model are shown to be more close to that of literature.

The parameter $G$, in equation (5), relates the stress tensor to the elongation parameters of the polymer. The presence of this parameter stabilizes the computations. In the case of Oldroyd fluid, the computations become less stable and higher Deborah numbers ( $D e>0.8$ in our case) cannot be reached.

Figure 2 shows the down-stream instability zone for Newtonian fluid and viscoelastic PTT fluids at $R e=100$ and $D e=0.5$. The addition of polymer particles to a Newtonian fluid stabilizes the flow which leads a narrower less extended instability zone. This effect can be explained by the postulate of McKinley et al. [12] who proposed that the destabilizing mechanism is a combination of streamline curvature and elastic normal stresses. These results agree with the findings of Sahin \& Owens [13] who treated the case of confined cylinder.

As the elastic effect stabilizes the flow, the pressure drop behind the cylinder decreases leading to of the of averaged base pressure coefficient with the increase of Deborah number. Also, the frequency of flow oscillation, non-dimensionalized in the form of Strouhal number, decreases. Figure 3 represents the change of averaged base pressure coefficient and Strouhal number versus the Deborah number for $\mathrm{Re}=$ 100. These results fairly agree with the work of Phan-Thien \& Dou [6] and that of Pipe \& Monkewtiz [3].

\section{CONCLUSION}

The incompressible two-dimensional laminar flow of viscoelastic fluid past a cylinder was simulated. The constitutive equation governing the viscoelastic behavior allowed to use PTT and Oldroyd-B models. The governing equations represented in generalizd orthogonal coordinates are descritized using finite volume method. Results showed that the PTT model is more stable in computations than the Oldroyd$B$ model. The viscoelastic effect stabilized the flow and decreased the drag and the oscillation frequency. 


\section{REFERENCES}

1 R.P. Chhabra, J.F. Richardson, Non-Newtonian Flow in the Process Industries, Butterworth-Heinemann, Oxford, 1999.

2 Cadot O. \& Lebey M., "Shear instability inhibition in a cylinder wake by local injection of a viscoelastic fluid", Physics of Fluids Vol. 11(2), 1999

3 Pipe C.J., Monkewtiz P.A., "Vortex shedding in flows of dilute polymer solutions", J. Non-Newtonian Fluid Mech. 139, 2006, pp. 54-67

4 Dou H. \& Phan-Thien N., "Parallelisation of an unstructured finite volume code with PVM: viscoelastic flow around a cylinder", J. Non-Newtonian Fluid Mech., 77, 1998, pp. 21-51

5 Dou H. \& Phan-Thien N., "The flow of an Oldroyd-B fluid past a cylinder in a channel: adaptive viscosity vorticity (DAVSS- $\omega$ ) formulation", J. Non-Newtonian Fluid Mech. 87, 1999, pp. 47-73

6 Phan-Thien N. \& Dou H., "Viscoelastic flow past a cylinder: drag coefficient", Comput. Methods Appl. Mech. Engrg. 180, 1999, pp. 243-266

7 Pope S. B, "The calculation of turbulent recirculating flows in general orthogonal coordinates", J. Comp Phys 2, 1978, pp. 197-217

8 Mompean G. \& Deville M., "Unsteady finite volume simulation of Oldroyd-B fluid through a three-dimensional planar contraction", J. Non-Newtonian Fluid Mech., 72, 1997 , pp. 253-279

9 Kamal H., Thais L., , Naji H. \& Mompean G. "Prédiction de l'écoulement laminaire bidimensionnel autour d'un cylindre, $8^{\text {ème }}$ Congrès de Mécanique (CDM 2007) El Jadida, Maroc du 17 au 21 Avril 2007

10 Williamson C. H. K., "Vortex dynamics in the cylinder wake", Annu. Rev. Fluid. Mech. (28), 1996, pp. 477-53

11 Oliveira P., " Method for time-dependent simulations of viscoelastic flows: vortex shedding behind cylinder", J. Non-Newtonian Fluid Mech. 101, 2001, pp. 113137

12 McKinley G. H., Pakdel P. \& Oztekin A., "Rheological and geometric scaling of purely elastic instabilities", J. Non-Newtonian Fluid Mech. 67, 1996 pp.19-47

13 Sahin M. \& Owens R. G., "On the effects of viscoelasticity on two-dimensional vortex dynamics in the cylinder wake", J. Non-Newtonian Fluid Mech. 123, 2004, pp. 121-139 
Table 1 Comparison of average drag coefficient with previous literature for $R e=100$

\begin{tabular}{|c|c|c|c|}
\hline De & Oldroyd $(\beta=0.5)$ & SPTT & Oliveira [11] \\
\hline 0.0 & 1.3806 & $1, r \vee \cdot \wedge$ & 1.3701 \\
\hline 0.5 & 1.4279 & 1.3732 & 1.3795 \\
\hline 1.0 & - & 1.3684 & 1.3732 \\
\hline 1.5 & - & 1.3644 & $\begin{array}{c}1.3630 \\
\text { (interpolated) }\end{array}$ \\
\hline
\end{tabular}

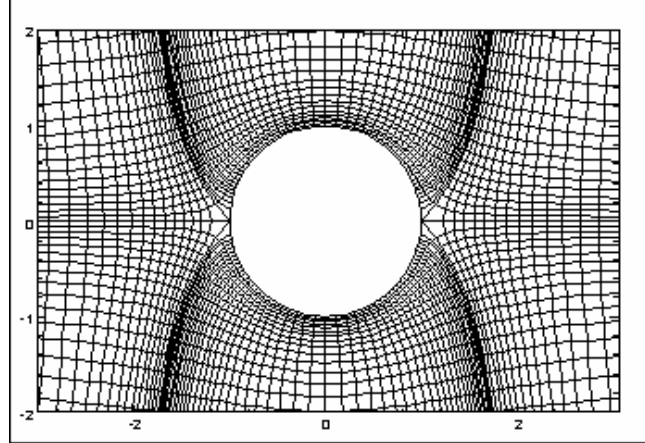

(a)

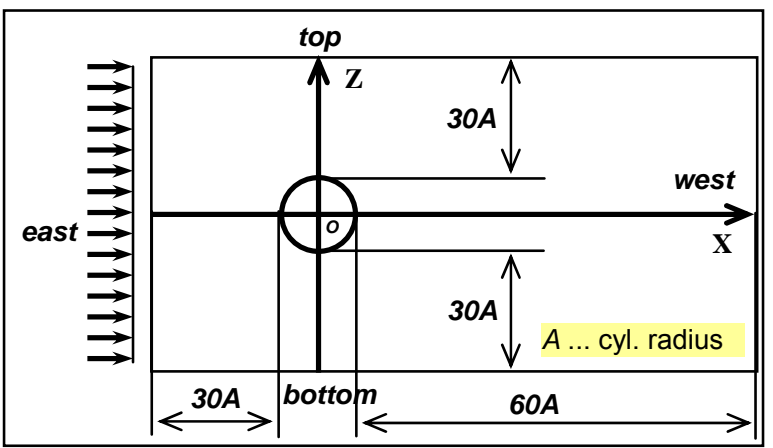

(b)

Fig. 1 (a) Zoomed view of the mesh around the cylinder, (b) domain limits.

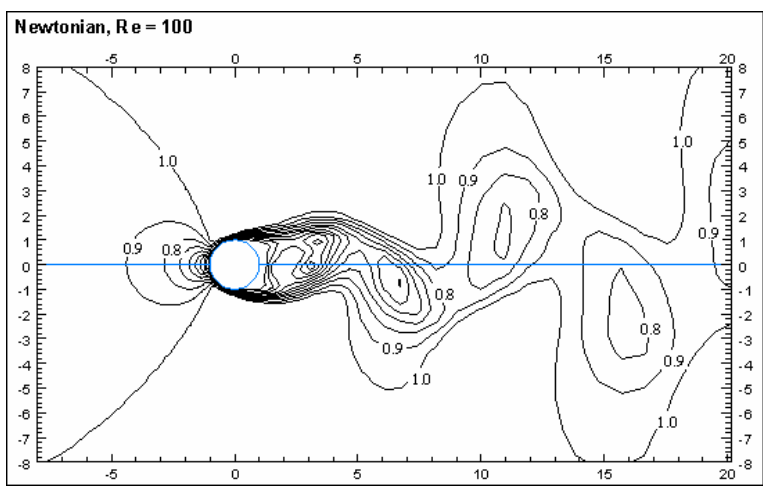

(a)

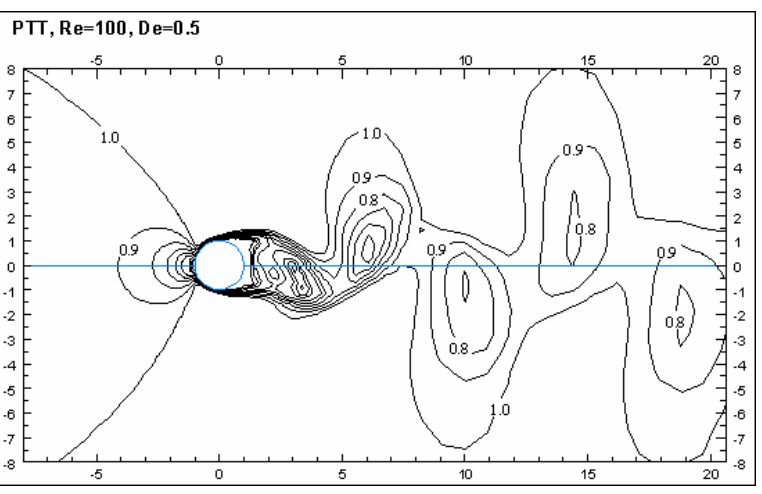

(b)

Fig. 2 Down-stream instability zone: (a) Newtonian fluid, (b) viscoelastic PTT fluid at $R e=100$ and $D e=0.5$.
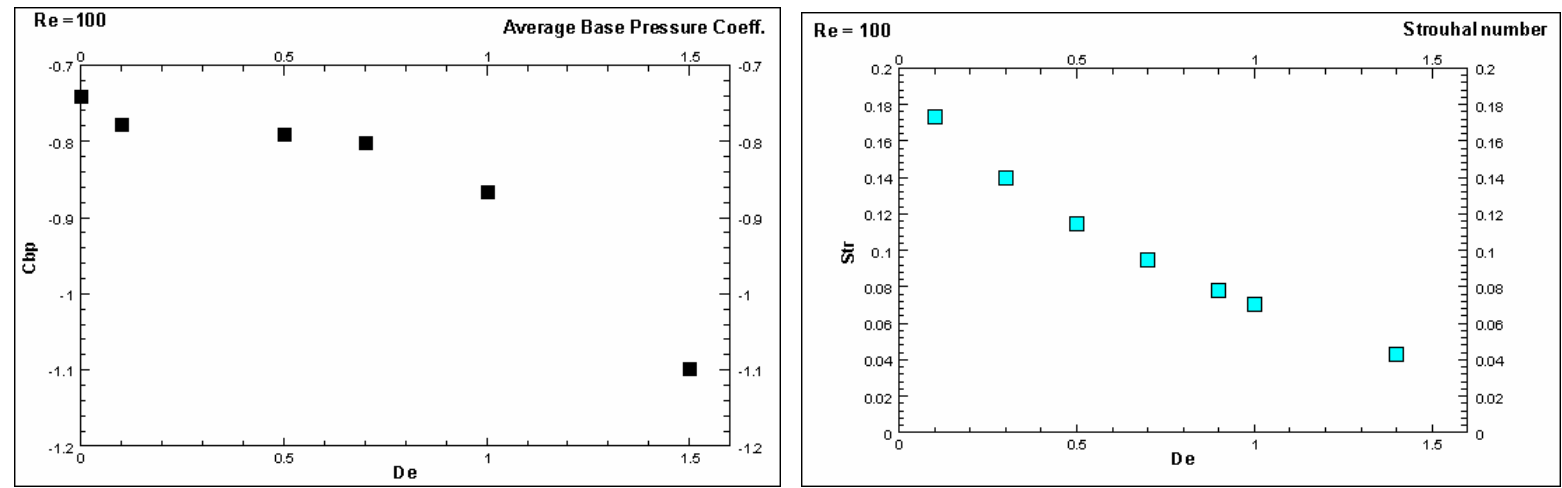

Figure 3 Averaged base pressure coefficient and Strouhal number versus the $D e(R e=100)$ 